\title{
ON A FUNCTIONAL EQUATION RELATED TO TWO-SIDED CENTRALIZERS
}

\author{
IRENA KOSI-ULBL
}

\begin{abstract}
The main aim of this manuscript is to prove the following result. Let $n>2$ be a fixed integer and $R$ be a $k$-torsion free semiprime ring with identity, where $k \in\{2, n-1, n\}$. Let us assume that for the additive mapping $T: R \rightarrow R$
\end{abstract}

$$
3 T\left(x^{n}\right)=T(x) x^{n-1}+x T\left(x^{n-2}\right) x+x^{n-1} T(x), \quad x \in R,
$$

is also fulfilled. Then $T$ is a two-sided centralizer.

In this paper $R$ will denote an associative ring with center $Z(R)$. For an integer $n>1$, a ring $R$ is said to be $n$-torsion free, if for $x \in R, n x=0$ implies $x=0$. The expression $x y-y x$ will be marked by $[x, y]$. The ring $R$ is prime if for $a, b \in R, a R b=(0)$ implies that either $a=0$ or $b=0$, and is semiprime if $a R a=(0)$ implies $a=0$. We indicate by $\operatorname{char}(R)$ the characteristic of a prime ring $R$. Let $X$ be a real or complex Banach space and let $\mathcal{L}(X)$ and $\mathcal{F}(X)$ denote the algebra of all bounded linear operators on $X$ and the ideal of all finite rank operators in $\mathcal{L}(X)$, respectively. An algebra $\mathcal{A}(X) \subseteq \mathcal{L}(X)$ is said to be standard if $\mathcal{F}(X) \subseteq \mathcal{A}(X)$. Let us point out that any standard operator algebra is prime, which is a consequence of HahnBanach theorem. An additive mapping $D: R \rightarrow R$ is called a derivation, if $D(x y)=D(x) y+x D(y)$ holds for all pairs $x, y \in R$. An additive mapping $D: R \rightarrow R$ is called a Jordan derivation if $D\left(x^{2}\right)=D(x) x+x D(x)$ is fulfilled

Received: 22.03.2017. Accepted: 21.09.2017. Published online: 31.01.2018.

(2010) Mathematics Subject Classification: 16W10, 46K15, 39B05.

Key words and phrases: prime ring, semiprime ring, Banach space, standard operator algebra, left (right) centralizer, left (right) Jordan centralizer, two-sided centralizer. 
for all $x \in R$. Every derivation is a Jordan derivation. The converse in general is not true. A classical result of Herstein [9] asserts that any Jordan derivation on a prime ring with $\operatorname{char}(R) \neq 2$ is a derivation.

A short proof of Herstein theorem can be found in [6]. Cusack [8] has generalized the theorem to 2-torsion free semiprime rings (an alternative proof can be found in [4]). Beidar, Brešar, Chebotar and Martindale [1] also generalized it considerably. Generalizations of Herstein theorem are also presented in [7]. An additive mapping $T: R \rightarrow R$ is called a left (right) centralizer if $T(x y)=T(x) y(T(x y)=x T(y))$ holds for all $x, y \in R$. We call $T: R \rightarrow R$ a two-sided centralizer if $T$ is both a left and a right centralizer. If $T: R \rightarrow R$ is a two-sided centralizer, where $R$ is a semiprime ring with extended centroid $C$, then there exists an element $\lambda \in C$ such that $T(x)=\lambda x$ for all $x \in R$ (see [2, Theorem 2.3.2]). An additive mapping $T: R \rightarrow R$ is called a left (right) Jordan centralizer if $T\left(x^{2}\right)=T(x) x\left(T\left(x^{2}\right)=x T(x)\right)$ holds for all $x \in R$. Zalar [17] has proved that any left (right) Jordan centralizer on a 2-torsion free semiprime ring is a left (right) centralizer. Lately several authors investigated centralizers on rings and algebras. Some of the results can be found in [3, 11, 12, 13, 14, 15, 16].

Let us start with the following result proved by M. Brešar in [5].

TheOREM 1. Let $R$ be a 2-torsion free semiprime ring and let $D: R \rightarrow R$ be an additive mapping satisfying the equality

$$
D(x y x)=D(x) y x+x D(y) x+x y D(x)
$$

for all $x, y \in R$. Then $D$ is a derivation.

An additive mapping $D: R \rightarrow R$, where $R$ is an arbitrary ring, satisfying equality (1) for all $x, y \in R$ is called a Jordan triple derivation. One can easily prove that any Jordan derivation on an arbitrary 2 -torsion free ring is a Jordan triple derivation (see, for example, [6] for the details), which means that Theorem 1 generalizes Cusack's generalization of Herstein theorem.

Motivated by this result, Vukman and Kosi-Ulbl in [14] proved the following

TheOREM 2. Let $R$ be a 2-torsion free semiprime ring with extended centroid $C$ and let $T: R \rightarrow R$ be an additive mapping. Suppose that

$$
3 T(x y x)=T(x) y x+x T(y) x+x y T(x)
$$

holds for all $x, y \in R$. Then $T$ is of the form $T(x)=\lambda x$ for all $x \in R$ and some fixed $\lambda \in C$. 
Let $n>2$ be a fixed integer and let $y=x^{n-2}$ in (2). Then we obtain

$$
3 T\left(x^{n}\right)=T(x) x^{n-1}+x T\left(x^{n-2}\right) x+x^{n-1} T(x) .
$$

One of our main purposes is to investigate equation (3) for additive mappings $T: \mathcal{A}(X) \rightarrow \mathcal{L}(X)$, where $X$ denotes a Banach space over $\mathcal{F} \in\{\mathbb{R}, \mathbb{C}\}$, $\mathcal{L}(X)$ denotes the algebra of all bounded linear operators acting on $X$, and $\mathcal{A}(X)$ is a standard operator algebra.

TheOREM 3. Let $\mathcal{L}(X)$ be the algebra of all bounded linear operators on $X$ and let $\mathcal{A}(X) \subseteq \mathcal{L}(X)$ be a standard operator algebra, where $X$ is a Banach space over the real or complex field $\mathcal{F}$. Suppose that there exists an additive mapping $T: \mathcal{A}(X) \rightarrow \mathcal{L}(X)$ satisfying the equality

$$
3 T\left(A^{n}\right)=T(A) A^{n-1}+A T\left(A^{n-2}\right) A+A^{n-1} T(A)
$$

for all $A \in \mathcal{A}(X)$ and a fixed integer $n>2$. Then $T$ is of the form $T(A)=\lambda A$ for all $A \in \mathcal{A}(X)$ and some fixed $\lambda \in \mathcal{F}$.

In the proof of Theorem 3 we shall use the result below, see Vukman [11].

THEOREM 4. Let $R$ be a 2-torsion free semiprime ring and let $T: R \rightarrow R$ be an additive mapping satisfying

$$
2 T\left(x^{2}\right)=T(x) x+x T(x)
$$

for all $x \in R$. Then $T$ is a two-sided centralizer.

It should be mentioned that in the proof of Theorem 3 we will use some methods similar to those used by Molnár in [10.

Proof of Theorem 3. We start with equality (4). Let us first consider $A \in \mathcal{F}(X)$ and a projection $P$ such that $A=A P=P A$. Identity (4) with $A=P$ yields that

$$
T(P) P=P T(P)=P T(P) P .
$$

In equation (4) we set $A+\alpha P$ for $A, \alpha \in \mathcal{F}$, and obtain

$$
3 \sum_{i=0}^{n}\left(\begin{array}{c}
n \\
i
\end{array}\right) T\left(A^{n-i}(\alpha P)^{i}\right)=(T(A)+\alpha T(P))\left(\sum_{i=0}^{n-1}\left(\begin{array}{c}
n-1 \\
i
\end{array}\right) A^{n-1-i}(\alpha P)^{i}\right)
$$


(6) $+(A+\alpha P)\left(\sum_{i=0}^{n-2}\left(\begin{array}{c}n-2 \\ i\end{array}\right) T\left(A^{n-2-i}(\alpha P)^{i}\right)\right)(A+\alpha P)$

$$
+\left(\sum_{i=0}^{n-1}\left(\begin{array}{c}
n-1 \\
i
\end{array}\right) A^{n-1-i}(\alpha P)^{i}\right)(T(A)+\alpha T(P)) .
$$

Collecting all expressions with coefficient $\alpha^{n-1}$ from equation (6) and using (5), we arrive at

(7) $3 n T(A)=T(A) P+P T(A)+n A B+n B A+(n-2) P T(A) P$,

where $B$ stands for $T(P)$. Right multiplication of (7) by $P$ gives

(8) $3 n T(A) P=T(A) P+P T(A) P+n A B+n B A+(n-2) P T(A) P$.

Similarly we obtain

(9) $3 n P T(A)=P T(A) P+P T(A)+n A B+n B A+(n-2) P T(A) P$.

Combining (8) and (9) gives us

$$
T(A) P=P T(A),
$$

which reduces equality (7) to

$$
3 T(A)=T(A) P+A B+B A .
$$

Multiplying the above by $P$ from the right gives

$$
3 T(A) P=T(A) P+A B+B A .
$$

Combining (10) with 11) we get

$$
T(A)=T(A) P .
$$

From the above equality one can conclude that $T$ maps $\mathcal{F}(X)$ into itself. Using (12), equality (10) reduces to

$$
2 T(A)=A B+B A .
$$

Multiplying (13) from the right and from the left by $A$, respectively, gives

$$
2 T(A) A=A B A+B A^{2} \text { and } 2 A T(A)=A^{2} B+A B A,
$$


respectively. Going back to (6) and collecting all expressions with coefficient $\alpha^{n-2}$ gives

$$
\begin{aligned}
& 3 n(n-1) T\left(A^{2}\right)=2(n-1)(T(A) A+A T(A)) \\
& +2(n-2)(A T(A) P+P T(A) A)+(n-1)(n-2)\left(A^{2} B+B A^{2}\right) \\
& +(n-2)(n-3) P T\left(A^{2}\right) P+2 A B A .
\end{aligned}
$$

Using (12) the above equality simplifies to

$$
\begin{aligned}
2\left(n^{2}+n-3\right) T\left(A^{2}\right)=2( & 2 n-3)(T(A) A+A T(A)) \\
& +(n-1)(n-2)\left(A^{2} B+B A^{2}\right)+2 A B A
\end{aligned}
$$

Combining (14) and (15) we get

$$
2 T\left(A^{2}\right)=T(A) A+A T(A) .
$$

Therefore we have an additive mapping $T: \mathcal{F}(X) \rightarrow \mathcal{F}(X)$ satisfying equation (16) for all $A \in \mathcal{F}(X)$. Since $\mathcal{F}(X)$ is prime, by Theorem 4 we may conclude that $T$ is a two-sided centralizer on $\mathcal{F}(X)$. We continue our proof by showing that there exists an operator $C \in \mathcal{L}(X)$ such that

$$
T(A)=C A \quad(A \in \mathcal{F}(X)) .
$$

For any fixed $x \in X$ and $f \in X^{*}$ by $x \otimes f$ we denote an operator from $\mathcal{F}(X)$ defined by $(x \otimes f) y=f(y) x$ for all $y \in X$. For any $A \in \mathcal{F}(X)$ we have $A(x \otimes f)=((A x) \otimes f)$. Let us choose $f$ and $y$ such that $f(y)=1$ and define $C x=T(x \otimes f) y$. Obviously, $C$ is linear. Using the fact that $T$ is a left centralizer on $\mathcal{F}(X)$ we obtain

$$
\begin{aligned}
(C A) x & =C(A x)=T((A x) \otimes f) y \\
& =T(A(x \otimes f)) y=T(A)(x \otimes f) y=T(A) x, \quad x \in X .
\end{aligned}
$$

Therefore we have $T(A)=C A$ for any $A \in \mathcal{F}(X)$. Since $T$ is a right centralizer on $\mathcal{F}(X)$ we obtain $C(A P)=T(A P)=A T(P)=A C P$, where $A \in \mathcal{F}(X)$ and $P$ is an arbitrary one-dimensional projection. Therefore $[A, C] P=0$. Using the fact that $P$ is an arbitrary one-dimensional projection we get $[A, C]=0$ for all $A \in \mathcal{F}(X)$. This means $C$ commutes with all operators from $\mathcal{F}(X)$. In other words,

$$
C x=\lambda x
$$


is fulfilled for all $x \in X$ and some fixed $\lambda \in \mathcal{F}$. Combining the above equation with (17) it follows that $T$ is of the form

$$
T(A)=\lambda A
$$

for any $A \in \mathcal{F}(X)$ and some fixed $\lambda \in \mathcal{F}$. We want to prove that the same equality holds on $\mathcal{A}(X)$ as well. For this purpose we introduce a mapping $T_{1}: \mathcal{A}(X) \rightarrow \mathcal{L}(X)$ defined by $T_{1}(A)=\lambda A$. Let us investigate a mapping $T_{0}=$ $T-T_{1}$. One can easily find out that $T_{0}$ is additive, it satisfies (4) and $T_{0}(A)=0$ for all $A \in \mathcal{F}(X)$. We will prove that $T_{0}(A)=0$ for all $A \in \mathcal{A}(X)$ as well. Let us introduce an operator $S \in \mathcal{A}(X)$ defined by $S=A+P A P-(A P+P A)$, where $A \in \mathcal{A}(X)$ and $P \in \mathcal{F}(X)$ is an one-dimensional projection. From the definition of the operator $S$ it follows immediately that $S P=P S=0$ and $S-A \in \mathcal{F}(X)$. Thus we have $T_{0}(S)=T_{0}(A)$. So we can rewrite (4) as

$$
3 T_{0}\left(S^{n}\right)=T_{0}(S) S^{n-1}+S T_{0}\left(S^{n-2}\right) S+S^{n-1} T_{0}(S) .
$$

Using the above and the facts that $T_{0}(P)=0$ as well as $S P=P S=0$, we obtain

$$
\begin{aligned}
T_{0}(S) S^{n-1}+ & S T_{0}\left(S^{n-2}\right) S+S^{n-1} T_{0}(S)=3 T_{0}\left(S^{n}\right)=3 T_{0}\left(S^{n}+P\right) \\
= & 3 T_{0}\left((S+P)^{n}\right)=T_{0}(S+P)(S+P)^{n-1} \\
& +(S+P) T_{0}\left((S+P)^{n-2}\right)(S+P)+(S+P)^{n-1} T_{0}(S+P) \\
= & T_{0}(S) S^{n-1}+T_{0}(S) P+S T_{0}\left(S^{n-2}\right) S+S T_{0}\left(S^{n-2}\right) P \\
& +P T_{0}\left(S^{n-2}\right) S+P T_{0}\left(S^{n-2}\right) P+S^{n-1} T_{0}(S)+P T_{0}(S) .
\end{aligned}
$$

Since $T_{0}(S)=T_{0}(A)$, we actually have

$$
T_{0}(A) P+S T_{0}\left(A^{n-2}\right) P+P T_{0}\left(A^{n-2}\right) S+P T_{0}\left(A^{n-2}\right) P+P T_{0}(A)=0
$$

Setting $\alpha A$ for $A$ in (18), we obtain

$$
\begin{array}{rl}
\alpha\left(T_{0}(A) P+P T_{0}(A)\right)+\alpha^{n-2} & P T_{0}\left(A^{n-2}\right) P \\
& +\alpha^{n-1}\left(S T_{0}\left(A^{n-2}\right) P+P T_{0}\left(A^{n-2}\right) S\right)=0 .
\end{array}
$$

This implies that $T_{0}(A) P+P T_{0}(A)=0$. Multiplying by $P$ on both sides gives $P T_{0}(A) P=0$. Multiplying on the right gives $T_{0}(A) P=-P T_{0}(A) P=0$. Since $P$ is an arbitrary one-dimensional projection, one can conclude that $T_{0}(A)=0$ for any $A \in \mathcal{A}(X)$. In other words, we have proved that $T$ is of the 
form $T(A)=\lambda A$ for all $A \in \mathcal{A}(X)$ and some fixed $\lambda \in \mathcal{F}$. The proof of the theorem is complete.

CONJECTURE. Let $R$ be a semiprime ring with suitable torsion restrictions and let $T: R \rightarrow R$ be an additive mapping satisfying the equation

$$
3 T\left(x^{n}\right)=T(x) x^{n-1}+x T\left(x^{n-2}\right) x+x^{n-1} T(x)
$$

for all $x \in R$ and a fixed integer $n>2$. Then $T$ is a two-sided centralizer.

The result below proves the above conjecture in the case when $R$ has an identity element.

TheOREm 5. Let $n>2$ be a fixed integer and $R$ be a $k$-torsion free semiprime ring with identity, where $k \in\{2, n-1, n\}$. Let us assume that, for the additive mapping $T: R \rightarrow R$,

$$
3 T\left(x^{n}\right)=T(x) x^{n-1}+x T\left(x^{n-2}\right) x+x^{n-1} T(x), \quad x \in R,
$$

is also fulfilled. Then $T$ is a two-sided centralizer.

Proof. Let us start from equation (19). Using the same techniques as in Theorem 3, we obtain

$$
\begin{aligned}
& 2\left(n^{2}+n-3\right) T\left(x^{2}\right)=2(2 n-3) T(x) x+2(2 n-3) x T(x) \\
& \quad+\left(n^{2}-3 n+2\right) a x^{2}+\left(n^{2}-3 n+2\right) x^{2} a+2 x a x, \quad x \in R
\end{aligned}
$$

and

$$
2 T(x)=x a+a x, \quad x \in R,
$$

where $a$ stands for $T(e)$. Comparing the steps of the proof of Theorem 3 with the beginning of the proof of Theorem 5 we see that equations 20 and (21) correspond to equations 15 and 13 , respectively. In the procedure mentioned above we used the fact that $R$ is $n$-torsion free. According to 21 ) we obtain

$$
2 T\left(x^{2}\right)=x^{2} a+a x^{2}, \quad x \in R .
$$

Multiplying 21] by $x$ first from the right and then from the left side we get

$$
2 T(x) x=x a x+a x^{2} \quad \text { and } \quad 2 x T(x)=x^{2} a+x a x, \quad x \in R .
$$


Using 22 and 23 in 20 after some calculation we obtain

$$
x^{2} a+a x^{2}-2 x a x=0, \quad x \in R .
$$

In the above calculation we used the assumption that the ring $R$ is 2 and $(n-1)$-torsion free. Now let us rewrite the above equation in the form

$$
[[a, x], x]=0, \quad x \in R
$$

Putting $x+y$ in place of $x$ in $(24)$ we obtain

$$
[[a, x], y]+[[a, y], x]=0, \quad x, y \in R
$$

Putting $x y$ in place of $y$ in 25 we obtain

$$
\begin{aligned}
0 & =[[a, x], x y]+[[a, x y], x] \\
& =[[a, x], x] y+x[[a, x], y]+[[a, x] y+x[a, y], x] \\
& =x[[a, x], y]+[[a, x], x] y+[a, x][y, x]+x[[a, y], x] \\
& =[a, x][y, x], \quad x, y \in R,
\end{aligned}
$$

where we also used (24) and (25). Thus we have

$$
[a, x][y, x]=0, \quad x, y \in R
$$

Substituting $y$ with $y a$ in the above we obtain $[a, x] y[a, x]=0$ for all $x, y \in R$. Since $R$ is semiprime, it follows from the last equation that $[a, x]=0$ for all $x \in R$. This means that $a \in Z(R)$ and 21 reduces to $T(x)=a x, x \in R$, since $R$ is 2-torsion free. It follows immediately that $T$ is a two-sided centralizer, which completes the proof.

\section{References}

[1] Beidar K.I., Brešar M., Chebotar M.A., Martindale W.S., 3rd, On Herstein's Lie map conjectures. II, J. Algebra 238 (2001), 239-264.

[2] Beidar K.I., Martindale W.S., III, Mikhalev A.V., Rings with generalized identities, Monographs and Textbooks in Pure and Applied Mathematics 196, Marcel Dekker Inc., New York, 1996. 
[3] Benkovič D., Eremita D., Vukman J., A characterization of the centroid of a prime ring, Studia Sci. Math. Hungar. 45 (2008), 379-394.

[4] Brešar M., Jordan derivations on semiprime rings, Proc. Amer. Math. Soc. 104 (1988), 1003-1006.

[5] Brešar M., Jordan mappings of semiprime rings, J. Algebra 127 (1989), 218-228.

[6] Brešar M., Vukman J., Jordan derivations on prime rings, Bull. Austral. Math. Soc. 37 (1988), 321-322.

[7] Brešar M., Vukman J., Jordan $(\theta, \phi)$-derivations, Glas. Mat. Ser. III 26(46) (1991), $13-17$.

[8] Cusack J.M., Jordan derivations on rigs, Proc. Amer. Math. Soc. 53 (1975), 321-324.

[9] Herstein I.N., Jordan derivations of prime rings, Proc. Amer. Mah. Soc. 8 (1957), 1104-1110.

[10] Molnár L., On centralizers of an $H^{*}$-algebra, Publ. Math. Debrecen 46 (1995), 89-95.

[11] Vukman J., An identity related to centralizers in semiprime rings, Comment. Math. Univ. Carolin. 40 (1999), 447-456.

[12] Vukman J., Centralizers on semiprime rings, Comment. Math. Univ. Carolin. 42 (2001), 237-245.

[13] Vukman J., Identities related to derivations and centralizers on standard operator algebras, Taiwanese J. Math. $11 \overline{2007), 255-265 .}$

[14] Vukman J., Kosi-Ulbl I., An equation related to centralizers in semiprime rings, Glas. Mat. Ser. III 38(58) (2003), $\overline{253-261 .}$

[15] Vukman J., Kosi-Ulbl I., Centralisers on rings and algebras, Bull. Austral. Math. Soc. 71 (2005), 225-234.

[16] Vukman J., Kosi-Ulbl I., A remark on a paper of L. Molnár, Publ. Math. Debrecen 67 (2005), 419-421.

[17] Zalar B., On centralizers of semiprime rings, Comment. Math. Univ. Carolin. 32 (1991), 609-614.

Faculty of Mechanical Engineering

UNIVERSITY OF MARIBOR

Smetanova 17

2000 MARIBOR

Slovenia

e-mail: irena.kosi@um.si 